\title{
Interaktionsarbeit: Herausforderung für die arbeitswissenschaftliche Forschung
}

\author{
Anita Tisch ${ }^{1} \cdot$ Beate Beermann ${ }^{1}$ - Louisa Wünnemann' ${ }^{1}$ Armin Windel ${ }^{1}$ \\ Online publiziert: 23. Januar 2020 \\ (c) Der/die Autor(en) 2020
}

\section{Zusammenfassung}

Interaktionsarbeit, verstanden als Arbeit an und mit Menschen, hat in den letzten Jahren einen erheblichen Bedeutungszuwachs erfahren. Während verschiedene Berufsfelder mit interaktiven Tätigkeitsanteilen bereits empirisch untersucht und unterschiedliche Konzepte zu Interaktionsarbeit vorgelegt wurden, sind relevante Fragen der Wirkung von Interaktionsarbeit auf Sicherheit und Gesundheit bei der Arbeit bislang weniger betrachtet worden. Zur menschengerechten Gestaltung von Interaktionsarbeit erscheint aus arbeitswissenschaftlicher Perspektive die Entwicklung einer übergeordneten Systematik zu den Anforderungen und Folgen von Interaktionsarbeit sowie ein Überblick über das vorliegende, empirisch gesicherte Gestaltungswissen sinnvoll.

Das Ziel des nachfolgenden Beitrages besteht darin, die Forschung zur Interaktionsarbeit im Kontext von Sicherheit und Gesundheit bei der Arbeit zu reflektieren sowie die Möglichkeiten einer konzeptionellen Einordnung unterschiedlicher spezifischer Formen der Interaktionsarbeit und die sich daraus ergebenden Gestaltungserfordernisse zu diskutieren.

Praktische Relevanz Für die gesundheits- und persönlichkeitsförderliche Gestaltung von Interaktionsarbeit sind Erkenntnisse über die spezifischen Arbeitsbedingungen und Anforderungen sowie deren Auswirkungen für die Beschäftigten zentral. Dieser Beitrag schlägt einen Ansatz für eine branchen- und berufsübergreifende Klassifikation von Merkmalen der Arbeit vor, die für die menschengerechte Gestaltung interaktiver Tätigkeiten relevant sind und zeigt die dafür erforderlichen weiteren Schritte auf.

Schlüsselwörter Interaktionsarbeit · Interaktive Arbeit · Dienstleistungsarbeit · Kundenkontakt · Arbeitsgestaltung

Louisa Wünnemann

wuennemann.louisa@baua.bund.de

1 Bundesanstalt für Arbeitsschutz und Arbeitsmedizin,

Friedrich-Henkel-Weg 1-25, 44149 Dortmund, Deutschland 


\title{
Interactive (service) work: challenges for applied research
}

\begin{abstract}
In recent decades, various occupations characterized by interactive work have been empirically investigated. In addition, several conceptualizations of interactive work — defined as work involving people-have been developed. Despite those efforts, the question whether and how interactive work affects safety and health at work has hardly been considered. From an occupational science perspective, there is no systematic overview of the current debate.

Therefore, the article aims at shedding light on current debates on interactive work in the context of occupational safety and health in order to stress the general need for further applied research. This is done in two interrelated steps. First, interactive work is defined and differentiated from other forms of work. Second, a synopsis and systematic integration of previous research on interactive work and its consequences are carried out which allows for identifying central research gaps.

Practical Relevance Profound knowledge about the specific working conditions and occupational requirements as well as their effects on employees is relevant for designing interactive work in a way that it supports an employee's health and personality. This article reflects the current state of knowledge and elaborates on further research in order to promote the future development of recommendations for practice.
\end{abstract}

Keywords Interaction work $\cdot$ Interactive work $\cdot$ Service work $\cdot$ Social service science $\cdot$ Customer contact $\cdot$ Work design

\section{Arbeitswissenschaftliche Perspektive auf die Interaktionsarbeit}

Die Arbeit an und mit Menschen, häufig bezeichnet als Interaktionsarbeit, hat in den vergangenen Jahrzehnten in wissenschaftlichen und gesellschaftlichen Diskussionen an Bedeutung gewonnen. Als interaktive Tätigkeiten bzw. Interaktionsarbeit werden dabei Tätigkeiten bezeichnet, „,deren,Gegenstände ' keine Objekte, sondern andere Menschen sind, die es zu beeinflussen, beispielsweise auszubilden, zu beraten, zu heilen, zu bedienen, zum Kaufen zu veranlassen, zu unterhalten gilt" (Hacker 2010, S. 21 f.). Der Begriff Interaktionsarbeit umfasst somit alle Arbeitstätigkeiten, bei denen Beschäftigte an und mit anderen Menschen arbeiten. Dies umfasst sowohl Kundinnen und Kunden, Klientinnen und Klienten oder Patientinnen und Patienten, aber auch die soziale Interaktion zwischen Vorgesetzten und Mitarbeitenden sowie zwischen Kolleginnen und Kollegen (Böhle et al. 2015; Dunkel 2013). Diese Interaktion kann grundsätzlich direkt von Angesicht zu Angesicht erfolgen oder aber - wie es im Kontext des technologischen Wandels häufiger geschieht - durch unterschiedliche Medien digital vermittelt oder unterstützt werden und umfasst sowohl verbale als auch schriftliche, zeitlich synchrone oder asynchrone Kommunikation. Zentraler Gegenstand der Interaktionsarbeit bleibt aber auch dabei der Mensch, was Interaktionsarbeit zu einer besonderen Art der Arbeit, zu einer „Leistung eigener Art“ macht (Dunkel und Weihrich 2018, S. 201). Die Übertragbarkeit von vorliegenden Konzepten und Handlungsempfehlungen, z. B. zur menschengerechten Gestaltung von Produktionsarbeit, ist dabei nicht ohne weiteres möglich. Ein maßgeblicher Grund hierfür liegt darin, dass interaktive Tätigkeiten in vielen unterschiedlichen
Branchen und Berufen Teil der Arbeitsaufgabe sind. Dies macht die Entwicklung eines eigenständigen konzeptionellen Rahmens sowie die Ableitung spezifischer Gestaltungsanforderungen erforderlich, die über branchen- und berufsspezifische Analysen und Betrachtungen hinausgehen.

Die arbeitswissenschaftliche Forschung nähert sich in einer wachsenden Zahl theoretischer wie empirischer Beiträge der Arbeit an und mit Menschen (Baethge und Wilkens 2001; Ernst und Kopp 2011; Nerdinger 1999), wenngleich dabei häufig berufsspezifische Studien vorherrschen (Ernst und Zühlke-Robinet 2018) und noch immer vorwiegend monologische Tätigkeiten, wie zum Beispiel in der Produktion, stärker im Fokus stehen. Da die Bandbreite an Interaktionsarbeit über verschiedene Tätigkeitsbereiche hinweg groß ist, liegen derzeit sehr unterschiedliche Erkenntnisse $\mathrm{zu}$ den spezifischen Arbeitsbedingungen und Anforderungen an die Beschäftigten sowie deren Beanspruchungsfolgen vor. Hierin mag begründet liegen, dass die Erkenntnislage zu Ausprägungsformen von Interaktionsarbeit und deren spezifischen Wirkungszusammenhängen zu Gesundheit, Leistungsfähigkeit und Wohlbefinden der Beschäftigten derzeit noch fragmentiert erscheint und eine zusammenfassende Einordnung der Befunde fehlt. Möglicherweise ließ die Fokussierung auf die Spezifität, die Frage einer integrierenden Klassifizierungsmöglichkeit der Anforderungen auf der Metaebene bislang in den Hintergrund treten. Eine solche Klassifikation böte aber die Chance einer Beschreibung der Anforderungen - über spezifische Anwendungsfelder hinweg - auf der Basis zentraler Arbeitsbedingungsfaktoren, deren Zusammenhang zu Beanspruchungsfolgen belastbar definiert ist. Diese erscheint umso wichtiger, da davon auszugehen ist, dass interaktive Anforderungen in vielen Arbeitsbereichen aufgrund des Wandels der Arbeits- 
welt weiter zunehmen werden (DGB Index Gute Arbeit 2018) und diese veränderten Anforderungen branchen- und berufsübergreifend zeitnah menschengerecht zu gestalten sind. Wie der nachfolgende Abschnitt aufzeigt, erscheint es auf der Grundlage der Charakteristika der verschiedenen interaktiven Tätigkeiten dabei sinnvoll, dass eine integrierende Klassifikation nicht nur physische, sondern vor allem psychische Anforderungen berïcksichtigt. Daher soll im vierten Abschnitt, auf der Basis des im Projekt „Psychische Gesundheit in der Arbeitswelt - wissenschaftliche Standortbestimmung" (Rothe et al. 2017) entwickelten Referenzmodells zur Beschreibung psychischer Belastungsfaktoren, eine auf den Gegenstand der Interaktionsarbeit ausgerichtete Anpassung des theoretischen Rahmenmodells vorgenommen werden.

\section{Bedeutung und Entwicklung von Interaktionsarbeit}

Welche Bedeutung Interaktionsarbeit aktuell für viele Beschäftigte einnimmt, zeigt die Beschäftigtenbefragung DGB Index „Gute Arbeit“ (2018): Etwa zwei Drittel aller Beschäftigten berichten, dass sie sehr häufig oder oft im direkten Kontakt zu Kundinnen und Kunden oder vergleichbaren betriebsexternen Personengruppen stehen, also interaktive Tätigkeiten ausüben. Betrachtet man zusätzlich die arbeitsbezogene Interaktion am Arbeitsplatz mit Kolleginnen und Kollegen, berichten $89 \%$ der Beschäftigten ohne Führungsverantwortung und $96 \%$ der Führungskräfte davon, häufig mit anderen, auch betriebsinternen Personen beruflich kommunizieren zu müssen (BIBB/BAuA-Erwerbstätigenbefragung 2018, eigene Auszählungen). Bislang wurde Interaktionsarbeit vorrangig dem Dienstleistungsbereich zugeordnet. Die Daten zeigen aber, dass interne wie externe Interaktion auch außerhalb des tertiären Sektors, in praktisch allen Berufssegmenten und Branchen an Bedeutung gewinnt (DGB Index Gute Arbeit 2018). Dabei kann der direkte Kontakt zu Kundinnen und Kunden, Klientinnen und Klienten oder Patientinnen und Patienten die Kernaufgabe darstellen, wie etwa in der Beratung und Pflege, oder aber wie z.B. häufig in technischen oder handwerklichen Berufen eine Ergänzung zu anderen (Kern-)Aufgaben sein (vgl. auch Böhle et al. 2015). So ist Interaktionsarbeit auch im Produktionssektor, vor allem durch die Integration von Abstimmungsprozessen mit Kundinnen und Kunden, in Teams sowie vor- und nachgelagerten Bereichen, wie z.B. in hybriden Wertschöpfungssystemen, von großer Relevanz (Brandt et al. 2016; Koch 2010).

Da Interaktionsarbeit in ganz unterschiedlichen Tätigkeitsbereichen und Kontexten geleistet wird, unterscheiden sich die Charakteristika der Interaktion sowie die Anforderungen an die Beschäftigten. So divergiert beispielsweise das Ausmaß, in dem eine Dienstleistung an die Wünsche des Kunden angepasst werden kann oder wie lange und häufig mit einer bestimmten Person interagiert wird. Überdies kann die Arbeit an und mit Menschen sowohl eine physische als auch eine psychisch-emotionale Interaktion erfordern. Dementsprechend können Begrifflichkeiten und das Verständnis von interaktiven Tätigkeiten stark divergieren (z. B. Birken und Dunkel 2013; Tombeil et al. 2013). Diese Heterogenität und Komplexität des Themenfeldes sowie die nicht selten schlagwortartige Verwendung von Begrifflichkeiten stellen eine Herausforderung für die Forschung zur Interaktionsarbeit und die Ableitung von Gestaltungswissen dar.

Vor diesem Hintergrund ist es für die weitere arbeitswissenschaftliche Analyse und Erforschung von Interaktionsarbeit sowie die darauf aufbauende Entwicklung von Gestaltungsempfehlungen für die Praxis wünschenswert, einen konzeptionellen Rahmen zu beschreiben, der unabhängig von spezifischen Determinanten (wie z.B. der Berufszuordnung) eine generelle Klassifikation für die Ableitung von Gestaltungsanforderungen vorgibt.

Diese Herangehensweise käme damit einer zentralen Herausforderung zukünftiger Gestaltungserfordernisse nach, nämlich die zunehmende Dynamik der Erscheinungsformen von Belastungssituationen bei Interaktionsarbeit angemessen abzubilden und ihren Zusammenhang zu Gestaltungsanforderungen systematisch aufzuzeigen. Veränderungen, denen das Begriffsverständnis und die Ausrichtung der Forschung Rechnung tragen muss, ergeben sich z.B. durch

- den aktuellen technologischen Wandel: Gerade interaktive Tätigkeiten verändern sich durch digitale Technologien zum Teil sehr stark. Soziale Interaktion findet zunehmend digital vermittelt statt (z. B. über Email, WhatsApp oder Telefon) und Tätigkeitsbereiche für Interaktionsarbeit wandeln sich oder entstehen neu (z.B. Ahlers et al. 2018). Ebenso bietet der Einsatz neuer Technologien (wie z.B. automatisierte Medikamentenausgabe im Pflegebereich oder Chatfunktionen bei Kundenberatungen) Beschäftigten neue Chancen und Möglichkeiten - stellen sie aber auch vor neue Herausforderungen. Erste Studien deuten darauf hin, dass Anforderungen durch den technologischen Wandel sowohl steigen als auch abnehmen können (Holler 2017; Kirchner 2015; Meyer et al. 2019; Schöllgen und Schulz 2016).

- Globalisierung: Durch eine wachsende globale Vernetzung von Arbeitsprozessen finden Interaktionen zunehmend zeitlich asynchron statt oder aber erfordern das Arbeiten in den Rand- und Nachtzeiten (Amlinger-Chatterjee 2016). Außerdem erfordern inter- und transkulturelle Kooperationen neue interaktive Kompetenzen, wie etwa Vorurteilsfreiheit oder Ambiguitätstoleranz (Kholin und Blickle 2015). 
- gesellschaftlicher und arbeitsorganisatorischer Wandel: Waren und Dienstleistungen werden zunehmend rund um die Uhr nachgefragt (Backhaus et al. 2018). Damit ändern sich die Erwartungen an interaktive Tätigkeiten, wie z.B. an die Erreichbarkeit von Kundenbetreuern. Darüber hinaus erfordern neue Formen der Zusammenarbeit, agilere Organisationsformen sowie die projektgesteuerte Arbeit neue Formen und auch eine neue Intensität der Interaktion (Lauche 2008).

- demografiebedingte Veränderungen und zunehmende Diversität der Gesellschaft: Durch die steigende Lebenserwartung sowie die Alterung der Gesellschaft werden neue Dienstleistungen und Versorgungssysteme für ältere Menschen, aber auch längere Lebensarbeitszeiten erforderlich (z. B. Bütow et al. 2007). Immer neue Anforderungen an die Beschäftigten und deren Qualifizierung ergeben sich im Kontext von Migrations- und Inklusionsprozessen beispielsweise durch unterschiedliche Sprachen und Wertvorstellungen oder den zunehmenden Umgang mit Menschen mit Behinderungen (z.B. Tezcan-Güntekin 2015; Zanier 2015).

\section{Interaktionsarbeit als Forschungsgegenstand und als Herausforderung für die Arbeitsgestaltung}

Seit dem ersten Schwerpunktheft der Zeitschrift für Arbeitswissenschaft zum Thema Interaktionsarbeit im Jahr 1999 wurde zwischenmenschlichen Interaktionen im Kontext von Erwerbsarbeit in der gesellschaftspolitischen und wissenschaftlichen Debatte zunehmend Aufmerksamkeit geschenkt (Baethge und Wilkens 2001; Ernst und Kopp 2011; Ernst und Zühlke-Robinet 2018). In verschiedenen einschlägigen Publikationen zum Thema wurde dabei argumentiert, dass die Interaktionsarbeit eine zusätzliche Anforderung für die Beschäftigten darstellt, die gegenüber der Arbeit an und mit materiellen oder immateriellen Objekten über spezifische Charakteristika verfügt und dementsprechend eine besondere Arbeitsgestaltung erfordert (Böhle et al. 2015; Dunkel und Weihrich 2006, 2012; Hacker 2018).

Die Formulierung dieser Anforderungen an die verschiedenen arbeitsbezogenen Disziplinen, sich intensiver mit den Herausforderungen für Interaktionsarbeit auseinanderzusetzen, haben - befördert durch verschiedene Bekanntmachungen, insbesondere des Bundesministeriums für Bildung und Forschung sowie der Deutschen Forschungsgemeinschaft zu einer in den letzten Jahren wachsenden Zahl konzeptioneller wie empirischer Beiträge zur Arbeit an und mit Menschen beigetragen. Dennoch ist festzustellen, dass die absolute Anzahl an Studien, die sich explizit dem Thema
Interaktionsarbeit widmet, bislang noch relativ klein ist. $\mathrm{Zu}$ dem zeigt die Sichtung der wissenschaftlichen Diskussion zum Thema Interaktionsarbeit auch, dass eine systematische Kategorisierung im Hinblick auf spezifische Anforderungen auf der Basis dieses Begriffs bislang nur ansatzweise stattgefunden hat.

So konnten über die Suche in den psychologischen Datenbanken PsychArticles, PsychINFO, PSYNDEX und E-Journals (via Ebscohost) und der soziologischen Datenbank SSOAR anhand der Schlagwörter ,Interaktionsarbeit“ und ,*interaktiv* *Arbeit*“ OR ,*interaktiv* *Tätigkeit*“ insgesamt lediglich 85 Publikationen im Zeitraum 1999-2019 identifiziert werden (Stand 11/2019). Davon haben sich 73 Beiträge spezifisch mit zwischenmenschlichen Interaktionen im Kontext von Erwerbsarbeit befasst. In der Gesamtschau wird deutlich, dass eine Systematisierung der Arbeiten bislang nicht stattgefunden hat. Vielmehr lässt sich ableiten, dass die Arbeit an und mit Menschen häufig nicht in ihrer Spezifität sondern im Rahmen anderer Debatten diskutiert wird, wie etwa in Abhandlungen der Pflegeforschung oder zum Thema care work (siehe beispielsweise Theobald et al. 2013; oder England 2005), bezüglich der Anforderungen von Dienstleistungsarbeit (z.B. Birken und Dunkel 2013) oder der sociology of service work (z.B. Leidner 1993 oder Korczynski 2013), sowie auch im Diskurs um Emotionsarbeit (emotional labour) (siehe Hochschild 1983 oder Wharton 2009).

In der Zusammenschau weisen die bisherigen Erkenntnisse zu Interaktionsarbeit aber auch darauf hin, dass über verschiedene Tätigkeitsbereiche hinweg Kerndimensionen der Arbeit beschreibbar sind, die interaktionsspezifische Anforderungen an die Beschäftigten stellen (z.B. DGBIndex Gute Arbeit 2018; Zapf 2002). In diesem Zusammenhang sind spezifische, den Forschungsgegenstand prägende Konzepte für die Arbeit an und mit Menschen entwickelt worden. So beschreibt beispielsweise das Konzept der Interaktionsarbeit nach Böhle et al. (2015) die interaktionsspezifische Anforderungs- und Belastungssituation der Beschäftigten anhand der Kernmerkmale Emotionsarbeit, Gefühlsarbeit, Kooperationsarbeit und dem Subjektivierenden Arbeitshandeln. Während Emotionsarbeit (der Umgang mit den eigenen Gefühlen) und der Umgang mit Unwägbarkeiten (Subjektivierendes Handeln) stärker auf den bzw. die Handelnde selbst bezogen sind, beinhalten die Anforderungen der Gefühls- und Kooperationsarbeit stärker die dialogische Interaktion mit dem Gegenüber. Andere Ansätze stellen z. B. Abstimmungsprobleme zwischen Dienstleister und Dienstleistungsempfänger, wie das soziologische Konzept ,interaktiver Arbeit“" nach Dunkel und Weihrich (2006, 2010, 2012), oder handlungsregulatorische Prozesse in den Vordergrund, wie das arbeitspsychologische Konzept der ,dialogisch-interaktiven Erwerbsarbeit“" nach Hacker (2009). Hacker (2018) hebt hierbei hervor, dass insbe- 
sondere in der Interaktionsarbeit der Umgang mit gefährlichen oder belastenden Ereignissen häufig zur Arbeitsaufgabe gehört, was selbst durch eine vorrausschauende Arbeitsgestaltung nicht gänzlich ausgeschlossen werden kann. Man denke hier z. B. an gefährdende Situationen im Polizeialltag oder aber an den Umgang mit dem Tod in der Palliativmedizin. Deshalb nehmen seiner Auffassung nach individuelle Bewältigungsstrategien in der Interaktionsarbeit eine wichtige Rolle ein. Die arbeitswissenschaftliche Forschung entbindet dies jedoch nicht von dem Anspruch, verhältnispräventive Maßnahmen zur Gestaltung der Arbeitsbedingungen in den Blick zu nehmen und weiterzuentwickeln.

Zusammenfassend leisten die dargestellten Konzepte zur Arbeit an und mit Menschen wesentliche Beiträge zur Abgrenzung der Interaktionsarbeit von anderen, eher monologischen Tätigkeiten und zur Frage, welche Arbeitsanforderungen mit Interaktionsarbeit im Speziellen einhergehen. Um Arbeit an und mit Menschen aus einer ganzheitlichen Perspektive menschengerecht gestalten zu können, sind sowohl interaktionsspezifische als auch solche Arbeitsbedingungen und -anforderungen zu berücksichtigen, die sich aufgrund ihrer Auswirkungen auf Gesundheit, Leistungsfähigkeit und Wohlbefinden der Beschäftigten allgemein als gestaltungsrelevant erwiesen haben. Dabei kommt neben den eher tätigkeits- oder bereichsspezifischen Anforderungen, wie der Arbeitsumgebung, insbesondere den psychischen Anforderungen eine zentrale Bedeutung zu.

\section{Vorschlag für eine integrierende Klassifikation zur Interaktionsarbeit}

Die vorausgegangenen Abschnitte haben Charakteristika von Interaktionsarbeit aufgezeigt, die - nicht zuletzt aufgrund des Wandels der Arbeitswelt - zunehmend Bestandteil der Anforderungen in immer mehr Arbeitsbereichen werden (DGB Index Gute Arbeit 2018). Eine Betrachtung und Analyse der Interaktionsarbeit auf übergeordneter Ebene ist daher für eine branchen- und berufsübergreifende Einordnung der Befunde und Gestaltungsoptionen der menschengerechten Gestaltung von Interaktionsarbeit erforderlich. Es ist davon auszugehen, dass Interaktionsarbeit ganz zentral mit psychischen Anforderungen einhergeht. Deshalb wird als möglicher Ausgangspunkt für eine integrierende Klassifikation der Anforderungen und Ressourcen von Interaktionsarbeit das im Rahmen des Projektes „Psychische Gesundheit in der Arbeitswelt - wissenschaftliche Standortbestimmung“ (Rothe et al. 2017) entwickelte Konzept der Schlüsselfaktoren der Arbeitsgestaltung herangezogen. Die systematische Sichtung von Arbeitsbedingungsfaktoren, die entweder als Anforderungen oder aber als Ressource im Kontext psychisch belastender Arbeitssituationen wirken, hat in diesem Projekt gezeigt, dass

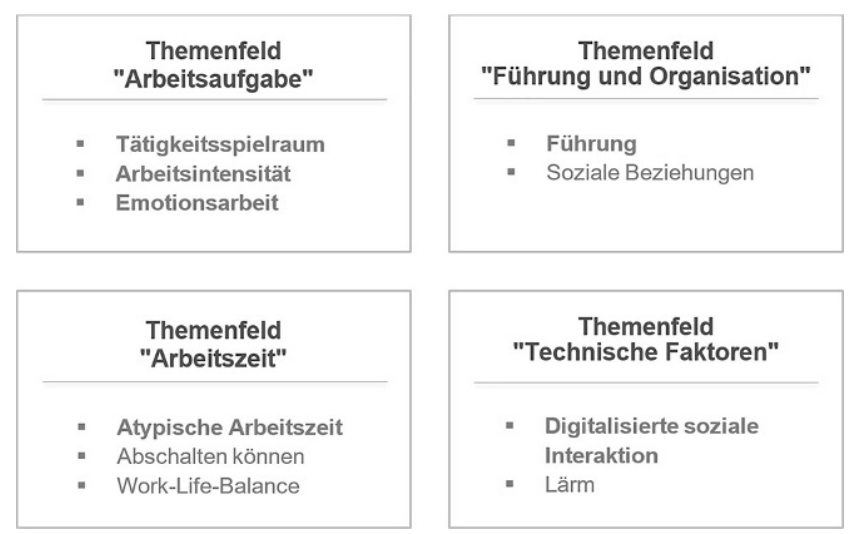

Abb. 1 Themenfelder und im Kontext von Interaktionsarbeit relevant erscheinende Arbeitsbedingungsfaktoren für die Arbeitsgestaltung auf Basis des BAuA-Projektes „Psychische Gesundheit in der Arbeitswelt"; fettgedruckt: Schlüsselfaktoren (in Anlehnung an Rothe et al. 2017).

Fig. 1 Potentially relevant working conditions in the context of interactive service work based on the project of BAuA entitle "mental health in the world of work" (translated); bold: key factors (cf. Rothe et al. 2017).

nicht allen Faktoren die gleiche „Strahlkraft“ zukommt. Vielmehr konnten tätigkeitsunabhängig Schlüsselfaktoren extrahiert werden, die über ihre eindimensionale Wirkung hinaus, d.h. in Kombination mit anderen Faktoren, wirksam werden. Auf der Basis vorliegender arbeitswissenschaftlicher Erkenntnisse zu Einflussfaktoren im Kontext psychisch belastender Arbeitsbedingungen haben Wissenschaftlerinnen und Wissenschaftler der Bundesanstalt für Arbeitsschutz und Arbeitsmedizin (BAuA) eine Vielzahl relevanter Arbeitsbedingungsfaktoren hinsichtlich ihres Zusammenhangs zu gesundheitsrelevanten Outcomes betrachtet. Die Arbeitsbedingungsfaktoren wurden dabei den Themenfeldern Arbeitsaufgabe, Führung und Organisation, Arbeitszeit sowie technischen Faktoren zugeordnet (Abb. 1). Diese können aufgrund ihrer zumeist förderlichen oder schädigenden Wirkung für Beschäftigte in Anlehnung an etablierte arbeitswissenschaftliche Modelle (z.B. Demerouti et al. 2001; Karasek 1979; Karasek und Theorell 1990) als Ressourcen und Stressoren klassifiziert werden. Auf der Basis von Scoping-Reviews ergaben sich - tätigkeitsunabhängig - systematische Einflüsse bezogen auf die Gesundheit, das Befinden und die Leistung von Beschäftigten.

Bezogen auf die psychische Belastung von Beschäftigten zeigte sich als eine zentrale Erkenntnis des Projekts, dass bestimmte Stressoren und Ressourcen eine Schlüsselrolle einnehmen. Diese üben einen starken Effekt auf andere Faktoren aus, werden selbst aber nur schwach beeinflusst. Schlüsselfaktoren im Sinne von Ressourcen ermöglichen den Beschäftigten mit Arbeitsanforderungen umzugehen, während die als Stressoren wirkenden Schlüsselfaktoren mit relevanten Beeinträchtigungen einhergehen. Wie 
auf Basis einschlägiger arbeitswissenschaftlicher Erkenntnisse zu erwarten ist, stellten sich als Schlüsselfaktoren im Themenfeld Arbeitsaufgabe der Handlungsspielraum, die Arbeitsintensität und die Emotionsarbeit heraus.

Betrachtet man nun die bisherige Forschung zu Interaktionsarbeit fällt auf, dass eben diese Faktoren gerade auch für die Interaktionsarbeit als bedeutsam hervorgehoben werden. So betonen Böhle et al. (2015) zunächst den Handlungsspielraum als zentrale Ressource für situatives und informelles Arbeitshandeln. Die Autoren zeigen, dass Handlungsspielräume gerade bei auftretenden Unwägbarkeiten, wie sie bei der Interaktion mit Menschen verstärkt vorkommen, als Ressource bei der Bewältigung der Anforderungen helfen können. Insbesondere zeitliche Handlungsspielräume, aber auch der emotionale sowie der Interaktionsspielraum haben sich dabei als relevant für eine menschengerechte Gestaltung von Interaktionsarbeit herausgestellt (Hacker 2018).

Sehr deutlich belegen Studien der letzten beiden Jahrzehnte, dass auch den Faktoren „Führung und Organisation“ und der „,sozialen Unterstützung“ eine Schlüsselfunktion als Ressource zukommen. Ihr Zusammenhang zur Interaktionsarbeit ist offensichtlich, da die Interaktion zwischen Führungskräften und Mitarbeitenden zu einem der am häufigsten untersuchten Konstrukte der Führungsforschung zählt und aktuell primär unter dem Aspekt differenziellen Führungsverhaltens und deren Wirkungen für die Mitarbeitenden diskutiert wird. Hier steht insbesondere auch die gesundheitsförderliche Führung als Ressource in der Diskussion, deren Erkenntnisse von Bedeutung für die menschengerechte Gestaltung interaktiver Tätigkeiten sind (Franke et al. 2015).

Während dem Handlungsspielraum wie auch der sozialen Unterstützung ein belastungsreduzierender Einfluss zugeschrieben werden kann, erweisen sich die Schlüsselfaktoren Arbeitsintensität und Emotionsarbeit als belastende Arbeitsbedingungsfaktoren. Dabei ist eine hohe Arbeitsintensität spätestens seit den 2000er Jahren branchenunabhängig zu beobachten (Lohmann-Haislah 2012). Die Arbeitsintensivierung betrifft aber gerade auch Bereiche, in denen überdurchschnittlich hohe emotionale Anforderungen und hohe Anteile interaktiver Tätigkeiten zu beobachten sind (vgl. zu Arbeitsintensität: Hirvonen und Husso 2012; Sonnentag et al. 2010; zu Emotionsarbeit: DGB Index Gute Arbeit 2018; Schöllgen und Schulz 2016; Zapf 2002).

Schließlich erweisen sich einige Belastungsfaktoren im Themenfeld Arbeitszeit als relevant. Im Vordergrund stehen die klassischen arbeitszeitgestalterischen Merkmale Dauer, Lage und Verteilung der Arbeitszeit und ihr Zusammenhang zu gesundheitlichen Beschwerden wie auch der Leistung von Beschäftigten. Aktuelle Studien zeigen auf, dass gerade Tätigkeiten mit hohem Anteil an Interaktionsarbeit geprägt sind von Wochenend- und Feiertagsarbeit wie auch von Arbeit in den Nacht- und Randzeiten (vgl. Backhaus et al. 2018). Damit kommt der Arbeitszeit sowohl bei der konzeptionellen Einordnung als auch bei der Gestaltung der Interaktionsarbeit hohe Bedeutung zu (Amlinger-Chatterjee 2016). Neben dem Belastungsaspekt hat die Gestaltung der Arbeitszeit aber auch maßgeblichen Einfluss auf die Erholung: Da in vielen sozialen Dienstleistungen Arbeitsinhalte und die Arbeitsverdichtung das Abschalten von der Arbeit erschweren (Becke et al. 2015), ist den Erkenntnissen zur Wirkung der Arbeitszeit auch bei der menschengerechten Gestaltung interaktiver Tätigkeiten besondere Aufmerksamkeit zu widmen.

Der Beschreibung der Arbeitsbedingungsfaktoren, die im Rahmen des Projektes der BAuA analysiert wurden und der Extraktion der Schlüsselfaktoren liegen keine tätigkeitsspezifischen Analysen zugrunde. Sie haben demzufolge einen generalisierbaren Aussagewert. Um nun aber diesen Ansatz zur Abbildung relevanter Anforderungen der Arbeitswelt auf die Spezifika der Interaktionsarbeit anzuwenden, ist im Detail zu prüfen, welche Bedeutung den identifizierten Schlüsselfaktoren für die Analyse und Gestaltung von Interaktionsarbeit zukommt und welche tätigkeitsspezifischen Ergänzungen oder Anpassungen notwendig erscheinen. So finden Charakteristika der Kundenintegration, wie z.B. die Art der Zusammenarbeit (psychisch, physisch, emotional) oder das Ausmaß der Individualisierung einer Dienstleistung aktuell keine Berücksichtigung. Zudem erscheint eine umfassende Analyse der Arbeitsanforderungen bei Interaktionsarbeit, die über die dargestellten psychischen Belastungsfaktoren hinausgehend auch physische Dimensionen und Rahmenbedingungen der Arbeit einbezieht, sinnvoll.

\section{Forschungsbedarf und Ausblick}

Zum jetzigen Zeitpunkt ist der aktuelle Forschungs- und Wissenstand zur Interaktionsarbeit sowie deren Anforderungen an und Folgen für die Beschäftigten eher heterogen und auf spezifische Anwendungsfelder orientiert. Vor dem Hintergrund der zunehmenden Verbreitung von Interaktionsarbeit in der Arbeitswelt erscheint jedoch eine Systematisierung und Einordnung der Erkenntnisse, die die Ableitung von übergreifenden Handlungsoptionen für unterschiedliche Tätigkeitsbereiche ermöglicht, erforderlich. Notwendig ist dabei, die Erkenntnisse und Befunde aus angrenzenden, auch internationalen Diskursen (wie z.B. um care work, emotional labour oder service work), die derzeit (noch) nicht konsequent unter dem Begriff der Interaktionsarbeit subsummiert werden, zu berücksichtigen und für die Gestaltung interaktiver Tätigkeiten zugänglich zu machen. Hierzu will das 2019 gestartete Projekt der BAuA und des Internationalen Instituts für Empirische Sozialökonomie (INIFES) „Interaktionsarbeit: Wirkungen 
und Gestaltung des technologischen Wandels (InWiGe)“ einen Beitrag leisten. Das Projekt hat dabei die Funktion eines wissenschaftlichen Begleitprogrammes im Rahmen des Förderschwerpunkts des Bundesministeriums für Bildung und Forschung ,Zukunft der Arbeit: Arbeiten an und mit Menschen“. Ein möglicher konzeptioneller Rahmen für die Erfassung der psychischen Arbeitsanforderungen, die nach erster Einordnung auch für interaktive Tätigkeiten relevant sind, wurde mit den Schlüsselfaktoren Arbeitsintensität, Handlungsspielraum, Emotionsarbeit, Arbeitszeit und Führungsverhalten/soziale Unterstützung umrissen. Um die Zielsetzung einer branchen- und berufsübergreifenden Kategorisierung und einer darauf basierenden Ableitung von für interaktive Tätigkeiten bedeutsamen Gestaltungserkenntnissen fortzusetzen, sind weitere relevante, z.B. auch physische Arbeitsbedingungsfaktoren, zu berücksichtigen.

Abgesehen von spezifischen Hinweisen zur Arbeitszeitgestaltung, die durchaus für die Interaktionsarbeit nutzbar sind, fehlen darüber hinaus für die Gestaltung der anderen Schlüsselfaktoren definierte und übertragbare Richtlinien und Grenzwerte. Vielmehr müssen Leitplanken der Arbeitsgestaltung für interaktive Tätigkeiten zum Teil von Grund auf neu erarbeitet werden. Dies setzt einen settingbezogenen, prozesshaften Ansatz voraus, in den alle betrieblichen Akteure einbezogen werden. Wünschenswert sind Dialogformen wie beteiligungsorientierte Workshops oder Gesundheitszirkel, die möglichst unter Einbeziehung von Expertinnen und Experten durchzuführen sind. Damit wird nicht nur die Compliance bei der Umsetzung der entwickelten Maßnahmen gestärkt, sondern auch die Gestaltungskompetenz der Mitarbeitenden erhöht. Eine zentrale betriebliche Anforderung wäre demzufolge die Entwicklung von Standards guter Prozessgestaltung, z. B. in Form Handlungsleitfäden. Gleichzeitig bedarf es für die betriebliche Umsetzung der Bereitstellung von Instrumenten und Verfahren, die die Spezifität des betrieblichen Kontextes erfassen und die Ableitung von Maßnahmen auf der betrieblichen Ebene ermöglichen. Hierzu erscheint die Entwicklung einer interaktionsarbeitsspezifischen Systematik bzw. Toolbox sinnvoll.

Förderung Open Access funding provided by Projekt DEAL.

Open Access Dieser Artikel wird unter der Creative Commons Namensnennung 4.0 International Lizenz veröffentlicht, welche die Nutzung, Vervielfältigung, Bearbeitung, Verbreitung und Wiedergabe in jeglichem Medium und Format erlaubt, sofern Sie den/die ursprünglichen Autor(en) und die Quelle ordnungsgemäß nennen, einen Link zur Creative Commons Lizenz beifügen und angeben, ob Änderungen vorgenommen wurden.

Die in diesem Artikel enthaltenen Bilder und sonstiges Drittmaterial unterliegen ebenfalls der genannten Creative Commons Lizenz, sofern sich aus der Abbildungslegende nichts anderes ergibt. Sofern das betreffende Material nicht unter der genannten Creative Commons Lizenz steht und die betreffende Handlung nicht nach gesetzlichen Vorschrif- ten erlaubt ist, ist für die oben aufgeführten Weiterverwendungen des Materials die Einwilligung des jeweiligen Rechteinhabers einzuholen.

Weitere Details zur Lizenz entnehmen Sie bitte der Lizenzinformation auf http://creativecommons.org/licenses/by/4.0/deed.de.

\section{Literatur}

Ahlers E, Berk B et al (2018) Digitalisierung: Dienstleistungsarbeit im Visier. Arbeitspapier der Hans-Böckler-Stiftung, Bd. 312

Amlinger-Chatterjee M (2016) Psychische Gesundheit in der Arbeitswelt. Atypische Arbeitszeiten. Bundesanstalt für Arbeitsschutz und Arbeitsmedizin, Dortmund

Backhaus N, Tisch A, Wöhrmann AM (2018) BAuA-Arbeitszeitbefragung: Vergleich 2015-2017. Bundesanstalt für Arbeitsschutz und Arbeitsmedizin, Dortmund

Baethge M, Wilkens I (2001) Die große Hoffnung für das 21. Jahrhundert? - Perspektiven und Strategien für die Entwicklung der Dienstleistungsbeschäftigung. Leske + Budrich, Opladen

Becke G, Wehl R, Wetjen A (2015) ,Die Kollegen sollen wissen, dass sie jederzeit einen Ansprechpartner haben“ - Interaktionsarbeit im Rahmen relationaler Handlungskoordinierung. In: Becke G, Bleses P (Hrsg) Interaktion und Koordination. Springer VS, Wiesbaden

BIBB/BAuA (2018). BIBB/BAuA-Erwerbstätigenbefragung 2018.

Birken T, Dunkel W (2013) Eine Bestandsaufnahme internationaler Literatur zu Service Science und Service Work. Arbeitspapier, Arbeit und Soziales, Bd. 282. Hans-Böckler-Stiftung, Düsseldorf

Böhle F, Stöger U, Weihrich M (2015) Interaktionsarbeit gestalten. Vorschläge und Perspektiven für humane Dienstleistungsarbeit

Brandt A, Polom L, Danneberg M (2016) Gute digitale Arbeit. Auswirkungen der Digitalisierung im Dienstleistungsbereich (WISODiskurs, 16). http://library.fes.de/pdf-files/wiso/12786.pdf. Zugegriffen: 29. Jan. 2018

Bütow B, Chassé KA, Hirt R (2007) Demographischer Wandel und soziale Dienstleistungen. In: Schmidt R (Hrsg) Soziale Arbeit nach dem Sozialpädagogischen Jahrhundert: Positionsbestimmungen Sozialer Arbeit im Post-Wohlfahrtsstaat. Barbara Budrich, Leverkusen, S 143-147

Demerouti E, Bakker AB, Nachreiner F, Schaufeli WB (2001) The job demands-resources model of burnout. J Appl Psychol 86:499-512

DGB-Index Gute Arbeit (2018) DGB-Index Gute Arbeit. Der Report 2018. Mit dem Themenschwerpunkt Interaktionsarbeit. Institut DGB-Index Gute Arbeit, Berlin

Dunkel W (2013) Arbeit und Interaktion. In: Hirsch-Kreinsen H, Minssen $\mathrm{H}$ (Hrsg) Lexikon der Arbeits- und Industriesoziologie. Edition sigma, Berlin, S 43-48

Dunkel W, Weihrich M (2006) Interaktive Arbeit - ein Konzept zur Entschlüsselung personenbezogener Dienstleistungsarbeit. In: Dunkel W, Sauer D (Hrsg) Von der Allgegenwart der verschwindenden Arbeit. Neue Herausforderungen für die Arbeitsforschung. Edition sigma, Berlin, S 67-82

Dunkel W, Weihrich M (2010) Arbeit als Interaktion. In: Böhle F, Voß G, Wachtler G (Hrsg) Handbuch Arbeitssoziologie. VS, Wiesbaden, S 177-200

Dunkel W, Weihrich M (Hrsg) (2012) Interaktive Arbeit. Theorie, Praxis und Gestaltung von Dienstleistungsbeziehungen. Springer VS, Wiesbaden

Dunkel W, Weihrich M (2018). Arbeit als Interaktion. In: Böhle F, Voß G, Wachtler G (Hrsg) Handbuch Arbeitssoziologie. Band 1: Arbeit, Strukturen und Prozesse (2. Auflage). Springer: Wiesbaden, S 201-230

England P (2005) Emerging theories of care work. Annu Rev Sociol 31:381-399

Ernst G, Kopp I (2014) Interaktionsarbeit als zentrales Element der Dienstleistungsinnovation. In: Schröder L, Urban HJ (Hrsg) Gute Arbeit. Bund-Verlag, Frankfurt/Main, S 338-348 
Ernst G, Zühlke-Robinet K (2018) Dienstleistungen - Wissenschaft und Forschung, Arbeit und Innovation. Nomos, Baden-Baden

Franke F, Ducki A, Felfe J (2015) Gesundheitsförderliche Führung. In: Felfe J (Hrsg) Trends der psychologischen Führungsforschung. Hogrefe, Göttingen, S 253-264

Hacker W (2009) Arbeitsgegenstand Mensch: Psychologie dialogischinteraktiver Erwerbsarbeit. Pabst Science, Lengerich/Berlin

Hacker W (2010) Psychische Regulation von Arbeitstätigkeiten. In: Kleinbeck U, Schmidt K-H (Hrsg) Arbeitspsychologie. Wirtschafts-, Organisations- und Arbeitspsychologie. Enzyklopädie der Psychologie, Bd. 1. Hogrefe, Göttingen, S 1-37

Hacker W (2018) Arbeitsgestaltung bei dialogisch-interaktiver Erwerbsarbeit. In: ver.di-Bereich Innovation und Gute Arbeit (Hrsg) Gestaltungskonzepte und Forschungsbedarf. Arbeit mit Menschen - Interaktionsarbeit humanisieren, Bd. 1. ver.di, Frankfurt am Main, S 31-35

Hirvonen H, Husso M (2012) Living on a knife's edge: temporal conflicts in welfare service work. Time Soc 21(3):351-370

Hochschild AR (1983) The Managed Heart. Commercialization of $\mathrm{Hu}-$ man Feeling. UC Press, Berkeley

Holler M (2017) Verbreitung, Folgen und Gestaltungsaspekte der Digitalisierung in der Arbeitswelt. Auswertungsbericht auf Basis des DGB-Index Gute Arbeit 2016. Institut DGB-Index Gute Arbeit, Berlin

Karasek R (1979) Job demands, job decision latitude, and mental strain: implications for job redisign. Adm Sci Q 24:285-307

Karasek R, Theorell T (1990) Healthy Work: Stress, Productivity and the Reconstruction of Working Life. Basic Books, New York

Kholin M, Blickle G (2015) Zum Verhältnis von Erwerbsarbeit, Arbeitswerten und Globalisierung. Eine psychosoziale Analyse. Z Arbeits Organisationspsychol 59(1):16-29

Kirchner S (2015) Konturen der digitalen Arbeitswelt. Eine Untersuchung der Einflussfaktoren beruflicher Computer- und Internetnutzung und der Zusammenhänge zu Arbeitsqualität. Kolner Z Soz Sozpsychol 47(4):763-791

Koch V (2010) Interaktionsarbeit bei produktbegleitenden Dienstleistungen. Am Beispiel des technischen Services im Maschinenbau. Dissertation, Universität Augsburg, Augsburg

Korczynski M (2013) The customer in the sociology of work: different ways of going beyond the management-worker dyad. Work Employ Soc 27(6): 1-7

Lauche K (2008) Neue Formen der Zusammenarbeit. In: BadkeSchaub P, Hofinger G, Lauche K (Hrsg) Human Factors. Psychologische Grundlagen sicheren Handelns in komplexen Arbeitsfeldern. Springer, Berlin, Heidelberg, S 205-234
Leidner R (1993) Fast Food, Fast Talk: Service Work and the Routinization of Everyday Life. UC Press, Berkeley

Lohmann-Haislah A (2012) Stressreport Deutschland 2012. Psychische Anforderungen, Ressourcen und Befinden, 1. Aufl. Bundesanstalt für Arbeitsschutz und Arbeitsmedizin, Dortmund

Meyer S, Tisch A, Hühnefeld L (2019) Arbeitsintensivierung und Handlungsspielraum in digitalisierten Arbeitswelten - Herausforderung für das Wohlbefinden von Beschäftigten? Ind Bezieh 2019(2):207-231

Nerdinger F (1999) Dienstleistung. In: Graf Hoyos C, Frey D (Hrsg) Arbeits- und Organisationspsychologie. Ein Lehrbuch. Psychologie Verlags Union, Weinheim, S 137-146

Rothe I, Adolph L et al (2017) Psychische Gesundheit in der Arbeitswelt - Wissenschaftliche Standortbestimmung, 1. Aufl. Bundesanstalt für Arbeitsschutz und Arbeitsmedizin, Dortmund

Schöllgen I, Schulz A (2016) Psychische Gesundheit in der Arbeitswelt - Emotionsarbeit. Bundesanstalt für Arbeitsschutz und Arbeitsmedizin, Dortmund

Sonnentag S, Binnewies C, Mojza EJ (2010) Staying well and engaged when de-mands are high: The role of psychological detachment. J Appl Psychol 95:965-976

Tezcan-Güntekin H, Breckenkamp J, Razum O (2015) Pflege und Pflegeerwartungen in der Einwanderungsgesellschaft. Sachverständigenrat Deutscher Stiftungen, Berlin

Theobald H, Szebehely M, Preuß M (2013) Arbeitsbedingungen in der Altenpflege. Die Kontinuität der Berufsverläufe - ein deutsch-schwedischer Vergleich. Z Arbeits Organisationspsychol 58(2):101-103

Tombeil A-S, Ganz W et al (2013) Dienstleistungsforschung - Trends, Themen, Entwicklungen aus internationaler Perspektive. MARS - International Monitoring of Activities and Research in Services - eine Bilanz. Fraunhofer Verlag, Stuttgart

Wharton AS (2009) The sociology of emotional labor. Annu Rev Sociol 35:147-165

Zanier G (2015) Altern in der Migrationsgesellschaft: Neue Ansätze in der Pflege - kultursensible (Alten-)Pflege und Interkulturelle Öffnung. In: Institut für Migrationsforschung und Interkulturelle Studien (Hrsg) focus Migration, Kurzdossier, August 2015, S 6-13

Zapf D (2002) Emotion work and psychological well-being. A review of the literature and some conceptual considerations. Hum Resour Manage Rev 12:237-268 